\title{
Les cadres des univers du récit et le patrimoine culturel
}

Narrations contemporaines chez les Nénetses

The Framing of Story Worlds and Cultural Heritage

Kertomusmaailmojen kehystys ja kulttuuriperintö

\section{Karina Lukin}

Traducteur : Christine Valla et Anne-Maj Karjalainen

\section{OpenEdition \\ Journals}

Édition électronique

URL : https://journals.openedition.org/efo/4082

DOI : $10.4000 /$ efo. 4082

ISSN : 2275-1947

\section{Éditeur}

INALCO

Édition imprimée

Date de publication : 1 janvier 2014

ISBN : 978-2-343-05394-3

ISSN : 0071-2051

\section{Référence électronique}

Karina Lukin, «Les cadres des univers du récit et le patrimoine culturel », Études finno-ougriennes [En ligne], 46 | 2014, mis en ligne le 14 octobre 2015, consulté le 08 juillet 2021. URL : http://

journals.openedition.org/efo/4082 ; DOI : https://doi.org/10.4000/efo.4082

Ce document a été généré automatiquement le 8 juillet 2021.

\section{(c) (7) (8)}

Études finno-ougriennes est mis à disposition selon les termes de la Licence Creative Commons Attribution - Pas d'Utilisation Commerciale 4.0 International. 


\title{
Les cadres des univers du récit et le patrimoine culturel
}

\author{
Narrations contemporaines chez les Nénetses \\ The Framing of Story Worlds and Cultural Heritage \\ Kertomusmaailmojen kehystys ja kulttuuriperintö
}

\section{Karina Lukin}

Traduction : Christine Valla et Anne-Maj Karjalainen

\section{NOTE DE L'AUTEUR}

Sources d'archives

MAC = Manuscripta Castreniana, Bibliothèque Nationale (Kansalliskirjasto).

1 «Le Nord n'a pas eu de chance ", a écrit en une formule lapidaire l'ethnographe et linguiste russe Nikolaj Vahtin dans son livre traitant de l'évolution des langues des populations du Nord de la Russie (Vahtin 2001, p. 250). Vahtin considère les peuples du Nord, leurs langues et leurs cultures traditionnelles comme les grands perdants de la modernisation de l'ère soviétique, bien qu'il ne partage pas les vues les plus pessimistes d'après lesquelles ces langues et ces traditions sont en voie de disparition. Durant l'ère soviétique la plupart des communautés linguistiques du Nord sont passées du bi- voire du plurilinguisme à la langue russe. Même dans le district autonome nénetse, comme dans de nombreux autres régions, le changement linguistique a eu lieu imperceptiblement et on n'a commencé à évoquer le processus en public qu'au moment de la perestrojka, voire même postérieurement (Menšakova 2003).

2 Au début des années 2000 je parvins à vivre au sein d'une communauté nénetse et j'ai pu constater que le nenetse de la toundra n'était utilisé comme moyen de communication quotidien que par une minorité. J'ai effectué des travaux de terrain auprès des habitants de l'île de Kolguev, située en mer de Barents, en quatre séjours sur la période 2000-2005. La durée de ces séjours a varié de deux semaines à trois mois, et 
leur objectif principal était de recueillir des traditions orales. J'ai mis un peu de temps à comprendre que les traditions orales se transmettaient également en russe, après quoi je me suis concentrée sur la collecte des productions langagières des habitants de l'île de Kolguev en russe. C'est pourquoi mes sources relèvent pour l'essentiel du folklore nenetse transmis en langue russe.

3 J'examine dans cet article les raisons pour lesquelles les Nenetses transmettent leur folklore en russe, ce qu'ils disent en russe et comment nous pouvons appréhender la signification de leurs récits. J'aborde en premier lieu la manière dont les Nenetses en sont arrivés à transmettre leurs traditions orales en russe et non dans leur langue d'origine, le nenetse de la toundra. Je traite ensuite de la signification des récits recueillis à l'aide des concepts d'univers du récit et de domaine de la narration et je les analyse également en relation avec les distances intertextuelles construites par ces récits. Je me suis concentrée sur la façon dont les traditions orales sont transmises dans le district autonome nenetse et, plus particulièrement, sur la façon dont les Nenetses habitant l'île de Kolguev transmettent leurs propres traditions. Je m'interrogerai sur les différences entre le mode de narration des Nenetses instruits et celui des autres habitants de l'île et sur la signification de ces différences. Les exemples que j'ai choisis concernent le chamanisme et permettent de traiter également dans l'article, de façon implicite, de l'évolution de la religiosité des Nenetses. L'analyse est toutefois essentiellement centrée sur l'utilisation de la langue dans une perspective plus générale liée à l'évolution linguistique.

\section{Les langues de l'île de Kolguev dans le contexte historique}

Comme Pertti Anttonen (2005 : 27-39) l'a établi, la tradition et sa disparition font partie des récits créés par la modernité. De façon pratiquement similaire Jim Wilce (2009) a montré que c'est la doxa contemporaine qui considère la mort de la tradition comme un processus totalement logique et inéluctable. Wilce admoneste les chercheurs qui tiennent ce discours et insiste sur le fait qu'en toile de fond du discours sur cette disparition inéluctable, on trouve souvent une suite d'événements historiques violents qui en sont les causes et qu'on évoque le plus souvent oralement et non par écrit. Je mettrai en évidence rapidement dans ce chapitre les causes historiques, sociales et culturelles qui ont amené les Nenetses de Kolguev à changer de langue. Mon intention n'est pas de faire verser des flots de larmes sur la mort de la tradition, mais d'examiner les raisons du changement de langue afin de mieux pouvoir traiter de la survie de la tradition orale après ce changement.

Il est important de noter que, dans l'histoire des Nenetses, ce ne sont pas seulement les liens avec les Russes, voire même la position dominante de ces derniers, qui ont provoqué la révolution qui devait amener les Nenetses à abandonner leur langue maternelle. Au contraire, les visiteurs étrangers à Kolguev ont décrit une situation de bilinguisme. Pour l'essentiel, cette révolution est liée aux activités communes des Nenetses et des Pomores, telles que le commerce et l'élevage des rennes, mais aussi au fait que la communication avec les popes d'origine allogène installés dans l'île se faisait en russe. Dès l'origine de leur installation dans l'île, les Nenetses de Kolguev ont été en contact avec des Russes, commerçants ou chasseurs pomores, et une grande partie d'entre eux sont entrés au service des Pomores pour l'élevage des rennes. La majeure 
partie des Nenetses est arrivée dans l'île à partir du milieu du XIX ${ }^{e}$ siècle. La décision de migrer dans l'île et toute la vie sur celle-ci sont basées sur une interaction étroite entre les communautés nenetse et russe (cf. Lukin 2012, p. 26-33).

6 Après la Révolution, le commerce et les autres échanges commencèrent à être soumis à des contrôles plus étroits, qui évoluèrent vers la construction d'une économie dirigée. Les Nenetses de Kolguev ont connu les mêmes transformations profondes de leur vie communautaire que les autres communautés vivant en URSS, puis en Russie (par exemple Slezkine 1994, p. 187-291, 337-351). Bien que la collectivisation de l'économie ait provoqué des changements indéniablement considérables sur les rapports de propriété entre Nenetses et Russes, ou entre Nenetses eux-mêmes, des bouleversements aux répercussions encore plus profondes eurent lieu après la collectivisation elle-même. Ces bouleversements, qui avaient pour la plupart débuté dans les années 1950, avaient pour but la transformation du socialisme en communisme, et ils correspondaient en pratique, dans le Nord, à des programmes de modernisation de la société. On regroupa les petites unités économiques pour en constituer de plus grandes, on offrit des services sanitaires dans les nouvelles agglomérations créées dans le Nord. Parallèlement, on commença à exploiter les ressources naturelles du Nord. Ce qui veut dire qu'aux fonctionnaires des services administratifs et culturels, les " priezžie $^{1}$ ", vinrent s'ajouter de nouvelles populations allogènes. Ces populations, qui comprenaient des Russes et des Ukrainiens, mais aussi des représentants de toutes les républiques de l'URSS, n'étaient pas préparées à la rencontre avec la différence culturelle. Les modes de vie différents et, en particulier, le mode de vie nomade, étaient globalement considérés comme inférieurs ou, pour le moins, jugés négativement (Grant 1995 ; Ljarskaja 2006 ; Sergeev 1955 ; Sokolova 1990 ; Tuisku 1999, p. 92-96, 161-162).

7 Beaucoup d'ethnographes de la période postsocialiste spécialistes des peuples du Nord mentionnent les internats comme un des principaux environnements où les attitudes négatives à l'égard de la culture des peuples autochtones ont été transmises. Le système scolaire de l'internat est rendu responsable de la perte de l'estime de soi des populations autochtones, de la désaffection des jeunes à l'égard du mode de vie traditionnel et de l'abandon de leur langue maternelle par les peuples du Nord. Les Nenetses eux-mêmes tiennent le système de pâturage alterné pour un facteur important qui a influé sur l'estime de soi et sur le discours des Nenetses de la toundra. Le système de pâturage alterné a été institué dans l'île de Kolguev à la fin des années 1970. Selon ce système, seuls les éleveurs travaillent par équipes qui se relaient dans la toundra, tandis que les femmes et les enfants s'installent au village. Ainsi, les différents membres de la famille vivent dans des endroits différents, les familles se disloquent et les savoir-faire exigeants de l'élevage des rennes et du nomadisme dans la toundra ne sont plus transmis aisément, voire plus du tout (Lukin 2012, p. 40-41; Tuisku 2001).

8 Les changements intervenus durant l'époque soviétique peuvent s'analyser comme la combinaison de la modernisation et du développement de la production industrielle. Selon Nikolaj Vahtin, les changements sociaux considérables qui ont affecté les peuples du Nord durant l'époque soviétique, outre le racisme, sont les facteurs majeurs qui ont accompagné le changement de langue. Bien que celui-ci ne se soit pas produit soudainement mais sur plusieurs décennies et soit toujours en cours, beaucoup de chercheurs ont situé son démarrage précisément à la fin des années 1950, ou avec les générations qui ont commencé à aller à l'école dans les années 1960. À cette époque, la 
langue nenetse de la toundra était en position de faiblesse dans le district autonome des Nenetses. On ne dispose pas pour cette époque de données statistiques fiables, ni de comparaisons avec d'autres régions, mais d'une vue d'ensemble donnée par le recensement de la population de 2002 qui montre que $32 \%$ seulement des Nenetses déclaraient avoir le nenetse de la toundra comme langue maternelle (Itogi 2004, p. 165). Des enquêtes linguistiques isolées ont également montré que, si la majeure partie de la génération des 40-50 ans connaît encore sa langue maternelle, la majorité des jeunes de 20 ans ne la connait plus. De plus, l'attitude de ces derniers à son égard est négative (Rokina 2003 ; Ermakov et al. 2003).

Dans le cadre de cet article, il est également essentiel d'attirer l'attention sur le fait que la dégradation du statut des langues du Nord a également affaibli leur utilisation en poésie et leurs capacités fonctionnelles. Dans la pratique, cela signifie qu'au pire la langue ne subsiste plus que dans les salutations, les événements culturels et les rituels de début et de fin des manifestations officielles ou publiques. Par exemple, dans le district autonome nenetse, ce sont essentiellement les parents nenetses qui savent transmettre le folklore en langue nenetse. De plus, la langue nenetse de la toundra, comme les manifestations du folklore, sont le passe-temps d'une élite culturelle ou font partie de leur travail et vivent essentiellement par la représentation en public. Globalement, le savoir traditionnel est donc passé du domaine des activités et des savoir-faire quotidiens à celui des paroles d'or des sages, comme cela s'est également produit chez d'autres peuples du Nord (Humphrey 1983, p. 230-231).

Toutefois, de nos jours, les traditions sont appréciées, les manifestations folkloriques se font en nenetse de la toundra et leurs acteurs sont très admirés. Plus particulièrement, les Nenetses apprécient les genres épiques, « sjudbabts ${ }^{2}$ » et « jarabts ${ }^{3}$ ». Ceux-ci ont dernièrement fait leur réapparition dans les concours de chant organisés à Nar'jan-Mar (cf. par exemple Divnye 2013), mais, dans l'ensemble, on ne trouve plus d'exécutant de ces chants encore en vie à l'ouest de l'Oural. Les chants individuels des Nenetses ( "sjo », « jabje"mja sjo ») sont également un genre toujours très prisé, de même que les individus qui connaissent les traditions en prose sont toujours appréciés dans les communautés. On dit que le chamanisme est en voie d'extinction dans la toundra à l'ouest de l'Oural, mais les chants rituels chamaniques sont toujours interprétés en tant que performances artistiques à l'occasion de manifestations publiques ou officielles. Dans l'ensemble, le chamanisme et la thématique qui lui est liée constituent aujourd'hui l'un des sujets préférés du discours oral des Nenetses. Ce point est étayé par les documents sur le chamanisme récemment parus dans le plus grand journal du district autonome nenetse, «Narjana Vynder» (par exemple Hanzerova 2013 ; Sjudbja 2013). La narration appréciée de nos jours regroupe des histoires et des récits dont le caractère est plus quotidien. Ils ont été étudiés de façon très superficielle et, de surcroit, les appellations des œuvres en prose varient considérablement d'une région à l'autre.

11 Durant la période où je travaillais sur le terrain, j'ai moi-même recueilli, entre autres, ce type de narration en prose. Les documents directs, génériques, en langue nenetse, qui me permettraient de faire des comparaisons et de fonder mon étude, me font malheureusement défaut. Sur la base de mes documents on peut cependant définir une communauté nenetse qui interprète activement tant des récits historiques et locaux que des récits personnels, en langue russe. Leur étude structurelle nécessiterait précisément les documents manquants en langue nenetse, ou encore un corpus plus 
large de récits traditionnels recueillis dans l'île de Kolguev. Faute d'une analyse structurelle, j'ai centré mon travail sur l'étude des rapports et des liens intertextuels. Ce type d'analyse a produit des résultats intéressants concernant la transmission des traditions orales dans le contexte d'un changement de langue.

Dans le contexte social post-soviétique, il ne faut pas oublier qu'il n'est pas judicieux de placer le mode et le contenu des discours des Nenetses dans le continuum de la tradition nenetse. Depuis longtemps, les Nenetses communiquent au-delà du niveau local, dans un contexte pluriculturel mais marqué par le monolinguisme, ou qui favorise le monolinguisme russe. Chez les Nenetses, les modes de représentation publique et culturelle ont pris forme durant l'époque soviétique, par interaction avec l'idéologie soviétique. Après la chute de l'URSS, les modes de représentation se sont multipliés et, de plus, les sujets qui étaient auparavant interdits - comme le chamanisme - sont devenus autorisés. Cependant, souvent, modes de représentation et contenus ont été conservés en l'état (cf. également Siikala \& Ulyashev 2012).

\section{Distances intertextuelles}

13 Bien que la narration en langue russe soit de nos jours monnaie courante chez les Nenetses occidentaux, le russe n'a pas complètement supplanté le nenetse en tant que langue de communication. Il y a une tension entre les deux langues, et divers groupes de Nenetses ont délibéré au sujet de cette tension de façon différente : beaucoup s'efforcent d'éviter de parler le nenetse dans les lieux publics mais, cependant, dans le cercle intellectuel de Nar'jan-Mar, la connaissance de la langue nenetse est appréciée. Les habitants de l'île de Kolguev utilisent encore le nenetse pour communiquer au sein de la famille, mais c'est le russe qui est utilisé dans les lieux publics, par exemple dans l'administration, à la poste ou dans les magasins, quoique, si besoin est, les services puissent être obtenus en langue nenetse. Contrairement à ce que l'on suppose en général, les éleveurs, qui passent le plus clair de leur temps dans la toundra, s'expriment en russe et non en nenetse. Car les Nenetses évoquent le passé et racontent des histoires en larges groupes, à l'occasion de rassemblements de famille ou de parentèle, ou de fêtes, où l'on se doit de parler russe.

Pour raconter des histoires ou se remémorer les temps anciens, les conteurs ou les écrivains disposent de différents moyens de communication et d'expression de la tradition. D'autre part, durant leurs performances, les conteurs utilisent pour exprimer la tradition des moyens variés, mais culturellement compréhensibles, qui influent sur l'interprétation qu'en fait le public. On peut exprimer les traditions par la parole ou par l'écrit, par exemple en employant une langue symbolique, en employant des moyens paralinguistiques, des formules et autres usages liés à la culture. Richard Bauman et Charles Briggs ont également montré que le conteur peut raccourcir ou rallonger la représentation et, de même, faire la distinction avec la tradition à l'aide de moyens variés. Selon Bauman et Briggs, interprètes et auditeurs participent ensemble à l'élaboration des liens intertextuels entre les textes représentés, de sorte que les représentations s'inscrivent dans des champs sémantiques plus larges et dans des réseaux de relations sociales (Bauman \& Briggs 1992, p. 149-163).

15 J'ai croisé ces conclusions de l'école de la performance avec les modèles d'analyse de Katherine Young - le domaine de la narration et l'univers du récit. L'argumentation de Young s'inscrit dans l'analyse des cadres de l'expérience de Goffman, mais aisément 
aussi dans le cadre plus large de l'anthropologie linguistique. Je ne pratique pas une analyse des cadres de l'expérience mais j'utilise les idées et les concepts de la théorie de Young. Par domaine de la narration Young entend l'événement narratif, c'est-à-dire le temps où la narration commence, en se constituant en enclave dans le cadre plus large du domaine de la conversation. Le domaine de la narration inclut dans l'événement les participants et leurs contributions orales. Cet événement mène le participant vers l'univers du récit ou vers ce qu'on raconte. L'autorité du conteur est le fondement de sa capacité à conduire les auditeurs du domaine de la conversation à celui de la narration puis à l'univers du récit, mais aussi à les ramener dans le cadre plus général de la communication. Ainsi un bon conteur est capable de se mouvoir entre divers domaines et univers, de sorte que le domaine de la narration comme l'événement qu'on raconte soient pertinents et signifiants dans le contexte plus large de la conversation (Young 1987, en particulier p. 22-60).

Young met en évidence les différences ontologiques entre domaine de la conversation, domaine de la narration et univers du récit qui permettent également de comprendre les règles $d u$ discours, de l'écoute et de l'interprétation. Selon Young l'univers du récit possède ses propres points de départ dans l'espace et dans le temps qui, dans le cadre de cet univers, sont aussi réels que ses personnages et leurs relations. Au domaine de la narration le conteur n'apporte qu'une partie de l'univers du récit, mais les auditeurs peuvent l'enrichir par leur imagination ou en se remémorant des écoutes, ou d'autres expériences, antérieures (Young 1987, p. 16, 189). Autrement dit, le domaine de la narration et le conteur délimitent l'univers du récit et n'en présentent dans le domaine de la narration que les parties importantes, intéressantes ou signifiantes à un moment donné. Les liens intertextuels se composent de séquences issues de savoirs ou d'expériences antérieurs en lien avec le domaine de narration du conteur et de l'auditeur.

De plus, les univers du récit interagissent les uns avec les autres et leurs traits distinctifs circulent d'un univers à l'autre. Les univers du récit traditionnel peuvent emprunter des traits aux univers du récit moderne, ou bien on peut mêler des traits de la vie quotidienne aux univers du récit de la croyance populaire. Les conteurs peuvent ainsi, à l'aide des traits de l'univers du récit, diminuer ou augmenter le lien intertextuel entre leur narration et la tradition. De la même manière les conteurs peuvent construire des liens ou des interfaces entre la tradition racontée en langue nenetse et en langue russe. Les conteurs construisent aussi des liens entre la tradition orale nenetse et les manières plus courantes de construire la réalité, au moyen desquels on peut circuler entre les différents genres dans la narration en langue russe. À l'aide des différents cadres, les conteurs construisent des relations entre le contexte de la conversation, le domaine de la narration et l'univers du récit. Dans la pratique ces cadres sont des méta-commentaires qui apportent des commentaires aux événements aussi bien de l'univers du récit que du domaine de la narration, à la portée de ces événements, etc.

\section{Purpej et le ver d'or}

Bien qu'à l'origine ce modèle d'analyse ait été conçu pour l'étude sur l'expression orale, on peut également l'utiliser pour l'étude des documents écrits. Dans ce cas, on peut prendre comme point de départ l'objectif plus vaste de l'ethnographie de la 
communication, qui consiste à examiner les dimensions symboliques et sociales des pratiques langagières. Les intellectuels de Nar'jan Mar publient un journal appelé Vyngy vada (Slovo tundry). Ce journal est rédigé en langue russe, bien qu'il traite des Nenetses et que, par ailleurs, il contienne essentiellement des articles traitant de sujets nenetses. Pour les raisons mentionnées ci-dessus, le nenetse ne pourrait pas être la langue de la publication, car seule une minorité de la population cible du journal serait alors capable de le comprendre. Le journal comprend une rubrique ayant pour titre Legendy $i$ mifi shamana Purpeja, "Des légendes et des mythes du chaman Purpej ». Elle est rédigée par Filipp Ardeev, professeur à la retraite, originaire de l'île Kolguev et résidant actuellement à Nar'jan-Mar. Ardeev est une personnalité qui apparaît fréquemment dans la vie publique du district et donne un visage à la culture, l'artisanat, la tradition orale des Nenetses ainsi qu'au chamanisme. Dans sa rubrique, Ardeev présente les récits qu'il a écrits sur le chamane le plus remarquable de l'île Kolguev.

Ardeev a lui-même enregistré des récits de son père en langue nenetse et envisage leur publication. En même temps, il reproduit ces mêmes récits en langue russe en les racontant entre autres aux touristes visitant le district et aux écoliers. Le texte paru dans le journal Vyngy vada, présenté ci-dessous, serait lui aussi basé sur un récit oral du père d'Ardeev, bien que cela ne soit pas mentionné dans le journal. En tant que texte, il est plutôt représentatif de la langue russe plus écrite que parlée, bien qu'il comporte de nombreuses références populaires. Le texte publié par Ardeev présente beaucoup de traits communs avec les récits que j'ai moi-même recueillis chez les Nenetses de l'île Kolguev. Aussi bien les récits que j'ai recueillis que ceux-ci sont caractérisés par un indice de base élevé de la distance intertextuelle avec la tradition en langue nenetse, puisque la tradition est contée ou rédigée en russe. Dans l'ensemble cependant, on peut augmenter ou diminuer cette distance dans les récits en langue russe, mais je n'approfondis pas dans cet article la réflexion sur la distance créée par la langue en soi.

Je présente ci-dessous un texte publié dans le journal Vyngy vada, qui se compose de deux parties, la première étant un genre d'introduction, et la deuxième présentant un récit plus ou moins délimité. Dans l'ensemble, on peut considérer que ces deux parties forment, dans le contexte du journal, un domaine ou un espace de narration menant vers l'univers du récit chamanique. Suit une brève partie qu'on peut décrire comme résolution. L'introduction a pour titre Skazy o velikom šamane Purpee, "Récits sur le grand chamane Purpej » :

Il y a longtemps, sur l'île Kolguev, vivait un chaman remarquable, Purpej. Encore aujourd'hui on raconte des histoires sur lui, et les vieilles générations de Nenetses évitent de prononcer le nom de ce grand sorcier à tort et à travers. C'était un chaman maigre et de petite taille. Des rennes, il n'en possédait pas tant que cela, un millier. Par contre, il n'avait pas son pareil sur la mer. Contrairement aux autres chasseurs qui allaient en mer, Purpej avait un grand bateau en bois, il connaissait tous les courants et manœuvrait une voile carrée. Souvent, allant à la chasse avec son ami Antipa, lui aussi chaman, ils pouvaient passer des jours entiers à naviguer entre les blocs de glace, dont ils se servaient également pour découper les mammifères marins qu'ils avaient chassés : morses, phoques barbus et marbrés ainsi que baleines.

Parfois les chamans se rendaient jusqu'à l'île Sengejskij, de l'autre côté du détroit, à environ 80 kilomètres de Kolguev. Parfois ils naviguaient près des plages de l'île, laissant alors leurs proies à un endroit précis sur les bancs de sable.

Purpej entretenait des relations commerciales avec des trappeurs norvégiens venus d'au-delà de la mer. Les habitants de l'île échangeaient avec les trappeurs norvégiens des peaux de renards des neiges, de renards roux et de mammifères 
marins, de la graisse et de la viande de renne, de la poudre, du plomb, du beurre et parfois des fusils. Le commerce se passait toujours de manière pacifique et amicale. Selon les habitants de l'île, ce grand chamane avait appris le chamanisme de son grand-père, dont plus personne ne se rappelle le nom. Le vrai nom de Purpej était Ivan Filippovič. Son surnom avait pour origine des cicatrices de variole. Ces cicatrices étaient comme de la rouille sur son visage (Purpej voulant dire rouillé).

Un jour le chaman reçut dans l'œil un jet de sang chaud d'un morse blessé, ce qui l'amena à perdre la vue d'abord progressivement, puis complètement. Mais il ne perdit pas ses pouvoirs...

21 Cette partie introductive est constituée d'abrégés ou de résumés des textes traitant de Purpej connus d'Ardeev. En même temps y sont présentées les principales bases ontologiques de l'univers du récit: le texte comporte des références à des univers du récit chamaniques et historiques. Dans le discours oral, ce genre d'introduction est typique : les parties introductives mènent les auditeurs - ou, comme dans ce cas, les lecteurs - vers l'univers du récit, mais elles participent aussi de cet univers. L'introduction fait également référence aux récits des habitants de l'île Kolguev, avec l'expression "selon les récits des habitants de l'île ${ }^{4}$ ». Cette expression inclut Ardeev dans la succession des narrateurs d'hier et d'aujourd'hui, tout en construisant une atmosphère de folklore. (Bauman 2004, p. 27-28). À la fin de cette partie introductive, Ardeev décrit l'apparence physique de Purpej et indique le nombre de rennes qu'il possède. Il s'agit là de la manière propre au folklore des Nenetses de présenter ses acteurs. Souvent les noms des personnages folkloriques révèlent certains de leurs traits de caractère ou certaines de leurs qualités : par exemple, les héros épiques peuvent avoir pour nom quelque chose comme "Chapeau de fer ", "Ski à sept brasses ", "Fer bouillant », "Taureau du maître » (Lehtisalo 1922, p. 86 ; Tereščenko 1980). Purpej est clairement indiqué comme étant un nom de chamane, et son nom laïc ou ordinaire est également mentionné dans la partie introductive. Le nombre de rennes ne témoigne pas uniquement de la richesse ou de la pauvreté du personnage, mais également de son statut social dans la communauté. Un troupeau de mille rennes représenterait dans la vie ordinaire des Nenetses une fortune relativement importante : ce genre de motifs exagératifs dirigent les interprètes du texte vers les univers narratifs traditionnels des Nenetses, dans lesquels les héros possèdent sans exception des troupeaux de mille têtes, ou au contraire, sont très pauvres. Par exemple, le poème épique syudbabts noté dans les années 1840 par M. A. Castrén commence de la manière suivante :

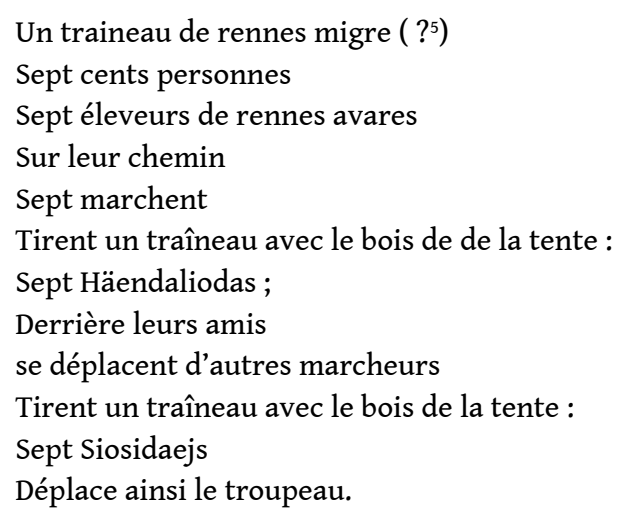

Dans cet exemple, au sein du groupe qui accompagne les troupeaux de rennes en traîneaux, les propriétaires des traîneaux de rennes, les Marodeats sont très riches alors que les Häendaliodas et les Siosidaejs, qui les suivent, sont pauvres: ils se déplacent à pied en tirant eux-mêmes le traîneau transportant le bois pour construire 
la tente. L'appellation des propriétaires peut se traduire littéralement par « les éleveurs de rennes avares » et Castrén ne l'a pas comprise comme un nom propre, même si à mon avis cela serait possible. À l'inverse, il a interprété comme noms propres les deux appellations qui suivent et explique dans ses notes que Häendalioda

peut signifier un arc qui se courbe légèrement dans la partie basse (хэндлё(сь) ‘se courber légèrement').

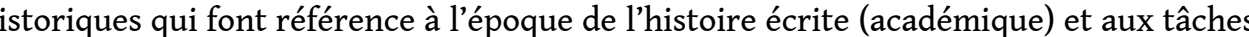
de la vie quotidienne. Il est dit, par exemple, que Purpej faisait du commerce avec d'autres chasseurs de mammifères marins. Ainsi, la partie introductive construit également l'image d'un homme qui a réellement vécu il y a quelques décennies, qui allait en mer avec le grand-père d'Ardeev et qui a appris ses connaissances de son propre père, un puissant chamane dont le nom est déjà oublié ${ }^{6}$. Les détails historiques peuvent également être vus comme faisant référence à des univers du récit originaux. Il s'agit des univers du récit de remémoration et des souvenirs spontanés des Nenetses, construits de la même manière que les univers des autres types de narration spontanée. La remémoration liée à la navigation, à la chasse aux mammifères marins et au commerce est très populaire chez les Nenetses de Kolguev. Même si d'autres Nenetses ont également chassé des mammifères marins, ce sont surtout ceux de Kolguev et, dans une moindre mesure, ceux de la Nouvelle Zemble, qui sont connus pour cela, et il s'agit là d'un élément fort de leur identité. Krupnik (1976) a lié la chasse aux mammifères marins à l'interaction entre les Nenetses et les chasseurs qui les précédaient dans la région; pour ma part, je verrais cela plutôt comme le résultat d'une interaction entre les Nenetses et les Pomores russes.

Après avoir pris connaissance de ces informations, le lecteur a donc déjà été introduit dans l'univers du récit chamanique, dans lequel les personnages nenetses sont capables d'actions que les personnes ordinaires ne peuvent accomplir. Les bases ontologiques de l'univers $\mathrm{du}$ récit sont, pour cette partie, claires, et Ardeev construit dans l'introduction un univers $\mathrm{du}$ récit très traditionnel, dont la différence avec les traditions chamaniques, si elle existe, est très limitée. En même temps, Ardeev construit également un univers du récit historique, en décrivant le chaman comme un véritable personnage historique, qui a réellement vécu avec les habitants de l'île Kolguev. Ces différents univers ne sont pas en contradiction entre eux, ils sont plutôt liés l'un à l'autre. 
Dans le récit, Purpej le chamane trouve un ver d'or, explique sa signification aux habitants de l'île et se l'approprie. Ardeev a donné au texte le titre suivant : « Purpej et le ver d'or ${ }^{7} »$ :

Un matin très tôt le chamane fut réveillé par des femmes effrayées. Elles étaient épouvantées par ce qu'elles voyaient : un ver sur le seuil de la tente. Il ne s'agissait pas d'un ver de terre ordinaire, mais d'un énorme ver doré. Même ses écailles diffusaient de la lumière. Tout le monde s'effraya en le voyant. Tout le monde, sauf Purpej. Il se saisit du ver, le roula comme un lasso et le plaça prudemment sous le seuil. Il raconta à tout le monde qu'un chamane hostile le lui avait envoyé. Le but de cet étrange ver était de pénétrer dans l'anus de Pourpeï et de le ronger de l'intérieur. Mais le grand vieillard s'avéra être plus puissant que le mal - ses esprits protégeaient leur maître de la destruction et d'une mort inévitable.

Plus tard, lors des déplacements, le chamane transportait lui-même le ver étrange et ne confiait cette tâche à personne d'autre. Tout à coup le ver disparut sans que personne sache où. Purpej n'en parla pas, et les gens n'osèrent pas lui demander.

Ce récit est un représentant exemplaire de l'univers du récit chamanique des Nenetses : les chamans s'y disputent entre eux par le biais d'esprits familiers apparaissant sous diverses formes. Le ver représente dans le récit l'une des formes de ces esprits. Dans l'univers du récit, le ver est présenté comme exceptionnel : grand et doré et, de cette même manière, le courage et l'ingéniosité de Purpej sont également présentés comme exceptionnels. Ceci est typique du mode de narration chamanique, dont j'ai traité les caractéristiques ailleurs (cf. Lukin 2011, p. 274-79 ; Lukin 2012). Dans le texte d'Ardeev, l'univers du récit est complet et entier : ses acteurs agissent conformément à ce qu'on attend de leurs rôles, sur lesquels est basée l'intrigue du récit. Le côté mystérieux des actes du chamane est conservé jusqu'à la fin du récit.

30 En comparant l'ensemble des récits d'Ardeev avec les récits que j'ai recueillis sur Kolguev, on peut constater que la plus grande différence réside dans le cadre qui définit les points de départ ontologiques de l'univers du récit. Cette différence ne concerne pas les univers du récit en soi, mais uniquement les manières dont se construisent les relations entre l'univers du récit, le domaine de la narration ainsi que les domaines de la conversation ou autres. Pour l'exprimer autrement, on peut également dire que la différence réside dans l'appréciation de l'univers du récit. (cf. Siikala 1984).

\section{Voyants et hypnotiseurs}

31 Le lien des habitants de l'île de Kolguev avec les pratiques chamaniques n'est pas aussi simple et facile que chez les intellectuels habitant les villes continentales. Leurs récits chamaniques ou teintés de chamanisme sont souvent empreints de scepticisme ou de doute. Autrement dit, les conteurs prennent leurs distances avec l'univers du récit chamanique et se tiennent aussi éloignés que possible du folklore chamanique (cf. Lukin 2012). La réalité se construit au travers du doute, ce qui est caractéristique des « remémorations ou, plus généralement, de la narration individualisée d'expériences personnelles » (Bennett 1999, p. 14-25). Dans les récits des habitants de l'île de Kolguev sur les chamanes, ceux-ci sont aussi tournés en ridicule. Bien qu'une telle dérision ait vraisemblablement été fréquente dans la période antérieure, elle est à mettre en relation, dans les récits contemporains, avec la propagande antireligieuse du temps de l'U.R.S.S. La dérision était un des outils privilégiés utilisés par la propagande et les conteurs de l'île de Kolguev paraissent l'avoir adoptée et introduite dans leur narration 
(Pospielovski 1988; Forsyth 1992, p. 287-289 ; Slezkine 1994, p. 219-228; Sundström 2007, p. 118-198).

De mon point de vue et de celui d'Ardeev, lorsque la plupart des intellectuels nenetses construisent un univers du récit chamanique et l'inscrivent dans le cadre de l'univers $\mathrm{du}$ récit historique, ils souhaitent souligner que cela s'est réellement produit. Mais, à l'inverse, les gens dits ordinaires de Kolguev établissent des rapports avec l'univers du récit chamanique et historique pour remettre en question l'action des chamans célèbres dans l'histoire. Il y a à cela plusieurs raisons, et l'une d'entre elles est certainement la propagande antireligieuse de la période soviétique. Une autre raison est à rechercher dans les traditions chamaniques elles-mêmes. Auparavant, dans les communautés chamaniques, les chamans avaient coutume de se cacher des étrangers ; les connaissances des chamans et leurs caractéristiques particulières étaient considérées comme un savoir interne à la communauté et étaient continuellement comparées. Les exemples qui suivent font clairement apparaître la tendance au doute dans la narration des habitants de l'île de Kolguev.

I(nformateur) : Le chamane est un homme ordinaire qui, tout simplement, connaît et sait pratiquer la magie. Étaient considérés comme chamanes les hommes qui savaient bien pratiquer l'hypnose. Un chamane peut, par exemple, être un voyant qui voit loin dans l'avenir, qui sait ce qui va arriver, et de tels hommes étaient tenus pour des chamanes. On dit toutefois, et cela s'est en fait produit plusieurs fois, qu'il voyait une semaine à l'avance. Et le chamane le dit à tous et tous le croient. Et, le moment venu, cela se passe effectivement. Le chamane peut donc, par exemple, dire ou simplement mettre en garde et les gens de cette sorte sont considérés comme des chamanes.

$\mathrm{K}$ (arina) L(ukin) : Bien. Vous dites que votre grand-père était un bon chamane...

$\mathrm{I}$ : Tout à fait. Il hypnotisait en effet les gens uniquement en les regardant dans les yeux. Il hypnotisait donc. Une fois, à l'occasion d'un mariage. Tout autour du lieu où on avait amené des rennes; il y avait des compétitions. J'ai moi-même participé personnellement à trois mariages; j'avais vu cela auparavant dans la toundra et c'était de grandes fêtes. Et alors notre grand-père montra que je sais. Il se frappa là avec son poignard et puis là d'un bout à l'autre et puis il prépara le traîneau. Et là il n'y avait qu'un harnais, avec un harnais seulement il tira le traîneau et avança avec le traîneau, et harnais et harnaché se soulevaient comme s'il y avait eu un renne, comme s'ils filaient autour de la tente avec un renne. Et les décorations du harnais étaient alors superbes. Ainsi allaient-ils bruyamment autour de la tente, sans renne mais absolument comme si des rennes avaient été attelés au traîneau, mais des rennes, il n'y en avait pas. Il a peut-être fait sept tours à toute vitesse en traîneau, et puis il a traversé et tiré ici et là où le traîneau le menait. Et c'est cela un chaman, il hypnotise, c'est de l'hypnose quand on est chaman.

Cet exemple est un extrait d'un entretien assez long avec un bon connaisseur des traditions des habitants de l'île de Kolguev. Déjà, avant cet entretien, mon informateur avait présenté les chamanes comme des hommes qui maitrisent les techniques qui permettent de berner les autres. Ils savent hypnotiser ou faire des tours de passe-passe. À noter aussi que j'ai supprimé au milieu de l'entretien un long passage où l'informateur compare les chamanes à des prestidigitateurs de cirque et évoque ses propres expériences dans les cirques. Bien que j'aie travaillé le style du texte afin qu'il soit plus accessible - notamment en supprimant les redites et les mots inachevés - cet extrait d'entretien apparaît clairement comme un énoncé en langue parlée et une narration orale. Cela ressort en outre des constructions de phrases imprécises et, par exemple, des expressions indexicales qui restent floues dans le texte initial. L'informateur montrait sa cage thoracique et la région de son abdomen en racontant 
pour indiquer où le chaman se frappait avec son poignard pour fixer le harnais au traîneau.

Compte tenu des coupures que j'ai effectuées dans l'extrait, l'introduction au récit cidessus est considérablement plus longue que l'introduction au récit transcrit, mais instaure également un cadre complètement différent. On souligne ainsi le caractère ordinaire du chamane et que la magie qu'il emploie n'est qu'une technique acquise. Le conteur souligne certes qu'on a fait confiance aux chamanes, par exemple en tant que voyants, et que leurs prédictions habituellement se réalisaient. Quand j'interroge le conteur au sujet de son grand-père, il fait évoluer les cadres de l'introduction vers l'univers du récit, à savoir un mariage à l'occasion duquel le grand-père souhaitait démontrer ses pouvoirs. Cette sorte de narration appartient à des traditions anciennes et s'inscrit dans la tradition tournant autour du chamanisme, dans le cadre de laquelle l'information sur les pouvoirs et les dons des chamans s'est diffusée dans la communauté. Les textes ethnographiques et les récits de voyages donnent à penser que les chamans font appel de façon répétée à des forces spirituelles ou de soutien moral, par exemple en se tailladant le corps. Castren, à ce sujet, a écrit :

Il y a quelques mois dans une tente de la toundra de Timan se réunirent trois Samoyèdes et un Russe. Un des Samoyèdes était initié aux secrets des «taadibe " (ou chamane en langue samoyède, du verbe qui signifie être en rut pour un cerf). Après être tombé dans une transe ordinaire, le chaman ordonna au milieu de la cérémonie que l'on se tire un coup de feu avec un fusil chargé. Un des deux autres Samoyède exécuta l'ordre mais la balle ne le toucha pas ou - comme on l'a dit rebondit sur son corps. Le fusil fut rechargé et le dernier Samoyède tira, à nouveau sans succès. Le Russe s'en étonna, chargea le fusil, tira et fit mouche. Le Samoyède mourut sur le champ. Dans la toundra de Kanin, j'ai rencontré un groupe de fonctionnaires envoyés pour enquêter sur l'affaire. Je ne connais pas les conclusions de l'enquête, mon récit est basé sur la rumeur publique (Castrén 1967, p. 140-141).

Clairement, mon informateur connaît bien cette tradition et son récit s'inscrit exactement et sans conteste dans cet univers du récit chamanique à travers lequel le chamane souhaite démontrer à la communauté son art et son pouvoir. Dans le récit, il réussit à étonner les invités du mariage en se harnachant lui-même et en tirant le traîneau autour de la tente. De même, dans cet univers du récit, le chamane et le chamanisme en général ne sont pas tournés en ridicule comme dans l'histoire entendue par Castrén dans les années 1840, où le chamane finit par être tué par la balle tirée par le Russe. Mais mon conteur conteste de l'extérieur les pouvoirs surnaturels du chamane et l'univers du récit fondé sur le chamanisme, tant dans l'introduction que dans ses appréciations, en répétant que les tours du chamane sont fondés sur l'hypnose. De même, les cadres du domaine de la narration semblent nier les hypothèses ontologiques de l'univers du récit et souligner les hypothèses ontologiques du contexte social régnant dans le domaine de la narration.

\section{Le discours de la tradition dans le Nord de la Russie au $x^{e}$ siècle}

Le contexte social plus vaste des deux récits présentés ci-dessus est celui du Nord de la Russie postsocialiste, où la narration en langue nenetse ne s'entend plus guère. Malgré cela, les contenus de la tradition intéressent aussi bien dans les communautés proprement nenetses qu'en dehors, surtout dans la ville de Nar'jan-Mar. Comme dans 
tous les districts du Nord de la Russie, en pays nenetse les peuples autochtones sont également présentés comme une altérité exotique qui donne son originalité à la région. À l'époque soviétique, les manifestations de cette originalité culturelle ont été développées en une vaste industrie culturelle, dont les acteurs passaient facilement des grands événements publics dans les centres administratifs aux petits événements locaux. L'héritage culturel propre à chaque groupe ethnique était donc présenté aussi bien à l'ensemble de la population soviétique qu'aux membres du groupe. L'héritage culturel à l'époque soviétique était sélectionné tant sur des critères esthétiques que politiques et consciemment promu comme une tradition conçue comme un spectacle; par exemple les thèmes religieux n'étaient pas traités, sauf sous un jour négatif.

Après l'effondrement de l'Union soviétique, cette tradition conçue comme spectacle a perduré en pays nenetse et, de plus, la culture a commencé à être présentée dans la presse, à la radio et à la télévision. L'esthétisme de nombreuses prestations reste similaire à celui de l'époque soviétique: les spectacles font référence à la culture matérielle et spirituelle prérévolutionnaire des Nenetses, par exemple par l'utilisation de costumes nationaux, de motifs du folklore nenetse ou de tentes. Néanmoins, les contenus des spectacles ne sont plus aussi fortement sélectionnés, et surtout la thématique relative au chamanisme a commencé à être fortement mise en évidence. Il s'agit clairement d'un thème exotique et intéressant, sur lequel beaucoup, surtout les étrangers, veulent en savoir davantage. Dans ce sens il peut être considéré comme un héritage culturel qui, dans des recherches récentes, a été défini comme un aspect sélectionné ou bien délimité et exposé de la culture, et dont la valeur en tant qu'héritage culturel est positive. En toile de fond des activités liées à l'héritage culturel, on trouve souvent la mise en scène d'une culture ou d'un groupe définissant les traits centraux de son identité. Ce genre d'activité a souvent été considéré comme réservé à une intelligentsia, mais il est important de constater que la population n'en faisant pas partie, autrement dit "le commun des mortels", elle aussi sélectionne, présente et évalue constamment sa propre culture et son discours (cf. Bendix 2000 ; Klein 2006).

Filipp Ardeev est devenu au sein du district la personne qui souvent, soit représente un chamane sur la scène, soit parle des chamanes aux étrangers. Ardeev a une connaissance personnelle de la tradition chamanique par le biais de ses parents et ses grands-parents, et, de plus, il a étudié la question à travers la littérature. En parlant du chamanisme ou de chamanes précis, il fait référence à la tradition orale qu'il connaît, comme dans l'exemple présenté ci-dessus. Cela lui donne incontestablement de l'autorité. L'appellation et les traits caractéristiques du chaman faisant référence à la tradition épique des Nenetses rapprochent la narration d'Ardeev de la tradition nenetse.

Son récit s'inscrit dans le cadre du réel mais, fortement aussi, dans celui des soutiens ontologiques à l'univers du récit chamanique. La narration ne crée pas seulement une image du chamane Purpej en tant que personnage historique ayant réellement existé, mais elle tend également à établir la véracité des bases métaphysiques du chamanisme, ce qui ne se produit que par exception dans les narrations des autres habitants de l'île de Kolguev. Les univers de leurs récits sont souvent aussi cohérents que celui d'Ardeev, mais les cadres de la narration nient souvent, de diverses manières, la véracité de la vision du monde chamanique. Dans l'exemple que j'ai présenté, l'ontologie chamanique est niée en évoquant l'hypnose, mais elle peut également l'être en affirmant que les 
chamanes étaient des illusionnistes ou des escrocs ${ }^{8}$, ou encore, plus implicitement, en tournant, par exemple, le récit en ridicule.

Dans les deux récits que j'ai présentés on peut donc constater des continuums avec la tradition nenetse, mais également avec la manière de la présenter à l'époque soviétique - les uns divergeant des autres. Le récit sur Purpej est typiquement celui d'un représentant de l'intelligentsia à l'époque soviétique, mettant en avant le caractère historique du chamanisme - il s'agit d'un phénomène que l'on raconte au passé. La nouveauté dans le récit d'Ardeev est l'appréciation positive du phénomène. Dans ce récit, comme dans d'autres productions des intellectuels du xxI siècle, le chamanisme est évoqué sous un jour positif, en tant qu'héritage culturel. Les chamanEs, et plus particulièrement ceux qui avaient de fortes personnalités, ceux qui étaient de bons commerçants et qui avaient d'autres capacités particulières, sont des personnages de leur ancienne culture que les intellectuels ont intérêt à mettre en évidence.

41 Les récits chamaniques des habitants de l'île Kolguev se positionnent, quant à eux, dans le continuum du discours athée de l'époque soviétique. Dans la propagande athée, les chamanes et les autres autorités religieuses étaient tournés en ridicule et méprisés, et on a essayé de diminuer leur autorité, notamment en montrant que leurs actions étaient basées sur l'escroquerie et sur diverses techniques psychologiques. Il est possible qu'un tel discours ait été adopté comme une manière de parler des chamanes réservée aux personnes étrangères, dont on peut considérer que je fais moi-même partie. C'est une manière sûre de transmettre de l'information sur les chamanes sans passer pour un partisan d'une métaphysique religieuse. Comme l'indique l'exemple extrait des journaux intimes de Castrén, la narration ayant pour but de nier le chamanisme a des racines plus profondes que la propagande athée de l'époque soviétique. Les chamans s'infligeant des souffrances par l'exercice de leur art, voire mourant du fait de leur vantardise, font partie d'une tradition chamanique.

\section{Conclusion}

42 J'ai mis en évidence dans cet article les différences qu'il y a au xxI ${ }^{\mathrm{e}}$ siècle entre les habitants ordinaires de l'île de Kolguev et l'intelligentsia en ce qui concerne leur manière de présenter la tradition orale, et plus particulièrement quand il s'agit de la tradition religieuse. La manière dont on parle du chamanisme et dont on raconte des histoires de chamans au niveau local est tendue et complexe : les images mentales et les idées athées de l'ère soviétique considérant la culture traditionnelle nenetse comme arriérée et blâmable ont été adoptées pleinement, pour le moins dans le discours. À l'inverse, les intellectuels ont l'habitude de parler du chamanisme avec aisance et même volontiers. Pour eux, il s'agit d'une part de l'héritage culturel nenetse qu'ils apprécient de manière positive. Pour l'intelligentsia, l'univers des récits chamaniques représente une sorte de réserve dans laquelle on peut puiser pour présenter les Nenetses aux visiteurs étrangers venus dans la ville de Nar'jan-Mar, ou pour enseigner quelque chose de la culture et des traditions s aux écoliers. En tant qu'héritage culturel, le chamanisme est un sujet exotique qui intéresse tout le monde.

Ces attitudes se retrouvent dans les cadres de la narration qui codifient le récit. Typiquement, les univers des récits présentés par les Nenetses sont homogènes, et leurs contenus remontent à la tradition orale des Nenetses et au chamanisme. Les chamans y apparaissent comme exceptionnels dans leur communauté en raison de 
leurs forces et de leur inventivité, mais ils possèdent également beaucoup d'autres qualités positives. Les univers du récit renvoient à des univers antérieurs, entendus ou compris par les Nenetses, ils remontent ainsi aux chants épiques comme aux récits en prose. $\mathrm{Au} \mathrm{XxI}{ }^{\mathrm{e}}$ siècle, on produit aussi des univers du récit en langue nenetse, mais la majeure partie est en langue russe, que les Nenetses communiquent entre eux ou qu'ils s'adressent à des étrangers arrivés dans la région. Il est cependant significatif que la tradition chamanique en elle-même, par exemple les chants rituels, n'est pas présentée en russe. Par conséquent, bien que la dissemblance intertextuelle entre les univers du récit et la tradition des Nenetses soit très faible au niveau du contenu, elle devient significative avec le changement de langue. On peut soit réduire, soit augmenter cette dissemblance à l'aide de cadres que les conteurs construisent dans le domaine de la narration. Les références aux autres motifs ou univers du récit chamanique et aux procédés de transmission de la tradition, ainsi qu'à la vie d'un chamane historique, tendent à réduire la dissemblance du récit avec la tradition orale nenetse. À l'inverse, en contestant l'activité du chaman, on augmente la distance avec le chamanisme et on en construit une image mentale ridicule.

Les deux types de cadres situent le chamanisme dans le passé et contestent son existence en tant que tradition vivante des Nenetses contemporains. De même, ils reflètent le bouleversement vécu par toute une communauté partageant une langue et une tradition, mais ils se positionnent de façon différente par rapport à la période antérieure à cette rupture.

\section{BIBLIOGRAPHIE}

ANTTONEN Pertti J., 2005, Tradition through Modernity. Postmodernism and the Nation-State in Folklore Scholarship, Studia Fennica Folkloristica 15, Helsinki: Finnish Literature Society.

BAUMAN Richard, 1977, Verbal art as performance, Illinois: Waveland Press.

BAUMAN Richard, 2004, A World of others' words: cross-cultural perspectives on intertextuality, Oxford: Blackwell Publishing.

BENDIX Regina, 2000, "Heredity, Hybridity and Heritage from One Fin de siècle to the Next", in Anttonen, Pertti J., Siikala, Anna-Leena, Mathisen, Stein R. and Magnusson, Leif (eds.), Folklore, Heritage Politics and Ethnic Diversity, A Festschrift for Barbro Klein. Botkyrka: Multicultral Centre, p. 37-54.

BENNETT Gillian, 1999, Alas, poor ghost! Traditions of belief in story and discourse, Logan, Utah: Utah State University Press.

BLOCH Alexia, 2004, Red Ties and Residential Schools: Indigenous Siberians in a Post-Soviet State, Philadelphia (PA): University of Pennsylvania Press.

BRIGGS Charles L. \& Bauman Richard, 1992, “Genre, Intertextuality, and Social Power”, Journal of Linguistic Anthropology 2(2), p. 131-172. 
CASTRÉN M. A., 1940, Samojedische Volksdichtung, Gesamm. von M. A. Castrén. Herausgegeben von T. Lehtisalo. Suomalais-Ugrilaisen Seuran Toimituksia LXXXIII. Helsinki : Suomalais-Ugrilainen Seura.

CASTRÉN M. A., 1967, Tutkimusmatkoilla pohjolassa, Suom. Joki, Aulis J. Helsinki :

Kustannusosakeyhtiö Tammi.

DIVNYE, 2013 = « ДИВНЫЕ МЕЛОДИИ ТУНДРЫ », НАРЬЯНА ВЫНДЕР 38. 11.4.2013.

Online http://nvinder.ru/article/vypusk-no-38-19950-ot-11-aprelya-2013-g/442-divnye-melodiitundry

ERMAKOv et al., 2003 = ЕРМАКОВ, « ВОСТРЕБОВАННОСТЬ РОДНОГО ЯЗЫКА НЕНЕЦКИМ НАСЕЛЕНИЕМ НАО. НЕКОТОРЫЕ ИТОГИ СОЦИОЛОГИЧЕСКОГО ОПРОСА », in МЕНШАКОВА, Е. Г. (РЕД.), МАТЕРИАЛЫ НАУЧНО-ПРАКТИЧЕСКОЙ КОНФЕРЕНЦИИ “НЕНЕЦКАЯ ПИСЬМЕННОСТЬ И РАЗВИТИЕ НАЦИОНАЛЬНЫХ ТРАДИЦИИ НАРОДА”, НАРЬЯН МАР : НЕНЕЦКИЙ ОКРУЖНОЙ КРАЕВЕДЧЕСКИЙ МУЗЕЙ, 181-183. FORSYTH James, 1992, A History of the Peoples of Siberia. Russia's North Asian Colony 1581-1990, Cambrige: Cambridge University Press.

GRANT Bruce, 1995, In the Soviet House of Culture. A Century of Perestroikas, Princeton: Princeton University Press.

HANZEROVA Irina, 2013 = ХАНЗЕРОВА ИРИНА, « СПОМНИ И СПОЙ ЖИЗНИ РОДА СВОЕГО », НАРЬЯНА ВЫНДЕР 26. 14.3.2013

Online http://nvinder.ru/article/vypusk-no-26-19938-ot-14-marta-2013-g/232-vspomni-i-spoyzhizn-roda-svoego

HUMPHREY Caroline, 1983, Karl Marx Collective. Economy, society and religion in a Siberian collective farm, Cambridge: Cambridge University Press.

ITOGI... 2004 = ИТОГИ ВСЕРОССИЙСКОЙ ПЕРЕПИСИ НАСЕЛЕНИЯ 2002 ГОДА (14 Т), ТОМ $4: 1$, НАЦИОНАЛЬНЫЙ СОСТАВ И ВЛАДЕНИЕ ЯЗЫКАМИ, ГРАЖДАНСТВО, МОСКВА : ФЕДЕРАЛЬНАЯ СЛУЖБА ГОСУДАРСТВЕННОЙ СТАТИСТИКИ.

KLEIN Barbro, 2006, "Cultural Heritage, the Swedish Folklife Sphere, and the Others", Cultural Analysis, 5, p. 57-80.

KRUPNIK I. I., 1976 = « СТАНОВЛЕНИЕ КРУПНОТАБУННОГО ОЛЕНЕВОДСТВО У ТУНДРОВЫХ НЕНЦЕВ », СЕВЕТСКАЯ ЭТНОГРАФИЯ, $2:$ 57-69.

KUPRIJANOVA Z.N., = КУПРИЯНОВА, З.Н., ЭПИЧЕСКИЕ ПЕСНИ НЕНЦЕВ, МОСКВА : НАУКА.

LEHTISALO T., 1922, Jurakkisamojedien lauluista. - Kalevalaseuran vuosikirja 2, p. 82-102.

LUKIN Karina, 2011, Elämän ja entisyyden maisemat. Kolgujev nenetsien arjessa, muistelussa ja kerronnassa, Helsinki : Suomalaisen Kirjallisuuden Seura.

LUKIN Karina, 2012, "Narrating the Last Shaman”, in Frog, Anna-Leena Siikala \& Eila Stepanova (eds.), Mythic discourses: studies in Uralic traditions, Helsinki: Finnish Literature Society, p. 355-379.

LJARSKAJA E. V., 2006 = ЛЯРСКАЯ, Е.В., « “У НИХ ЖЕ ВСЕ НЕ КАК У ЛЮДЕЙ ...”: НЕКОТОРЫЕ СТЕРЕОТИПЫ ПРЕДСТАВЛЕНИЯ ПЕДАГОГОВ ЯМАЛО-НЕНЕЦКОГО ОКРУГА О ТУНДРОВИКАХ ॥, АНТРОПОЛОГИЧЕСКИЙ ФОРУм forum, 13, 3-38.

MENŠAKOVA E. G. (red.), 2013 = МЕНШАКОВА, Е. Г. (РЕД.), МАТЕРИАЛЫ НАУЧНО-ПРАКТИЧЕСКОЙ КОНФЕРЕНЦИИ “НЕНЕЦКАЯ ПИСЬМЕННОСТЬ И РАЗВИТИЕ НАЦИОНАЛЬНЫХ ТРАДИЦИИ НАРОДА”, НАРЬЯН МАР : НЕНЕЦКИЙ ОКРУЖНОЙ КРАЕВЕДЧЕСКИЙ МУЗЕЙ, 181-183 
POSPIELOVSKY Dmitri, 1988, Soviet Antireligious Campaigns and Persecutions. Volume 2 of A History of Soviet Atheism in Theory and Practice, and the Believer, Hampshire and London: Macmillan Press.

ROKINA V. V., 2003 = РОКИНА, В.В., « НЕНЕЦКИЙ ЯАЫК : БЫТЬ ИЛИ ЗАБЫТЬ ? ПО ИТОГАМ КРУГЛОГО СТОЛА », in МЕНШАКОВА, Е. Г. (РЕД.), МАТЕРИАЛЫ НАУЧНО-ПРАКТИЧЕСКОЙ КОНФЕРЕНЦИИ “НЕНЕЦКАЯ ПИСЬМЕННОСТЬ И РАЗВИТИЕ НАЦИОНАЛЬНЫХ ТРАДИЦИИ НАРОДА", НАРЬЯН МАР : НЕНЕЦКИЙ ОКРУЖНОЙ КРАЕВЕДЧЕСКИЙ МУЗЕЙ, 132-135.

SERGEEV M. А., 1955 = СЕРГЕЕВ, М.А., НЕКАПИТАЛИСТИЧЕСКИЙ ПУТЬ РАЗВИТИЯ МАЛЫХ НАРОДОВ СЕВЕРА, МОСКВА : И.Э. ИМЕНИ МИКЛУХО-МАКЛАЯ

SIIKALA Anna-Leena, 1984, Tarina ja tulkinta, Helsinki : Suomalaisen Kirjallisuuden Seura.

SIIKALA Anna-Leena \& ULYASHEV Oleg, 2012, Hidden Rituals and Publiv Performances, Helsinki: Finnish Literature Society.

SLEZKINE Yuri, 1994, Arctic Mirrors. Russia and the Small Peoples of the North, Ithaca (NY): Cornell University Press.

SOKOLOVA Z.P., 1990 = СОКОЛОВА, З.П., « НАРОДЫ СЕВЕРА СССР : ПРОШЛОЕ, НАСТОЯЩЕЕ И БУДУЩЕЕ », СОВЕТСКАЯ ЭТНОГРАФИЯ 6, 17-32.

SUNDSTRÖM Olle, 2007, Kampen mot "schamanismen". Sovjetisk religionspolitik gentemot inhemska religioner i Sibirien och norra Ryssland. Studier av inter-religiösa relationer 40, Uppsala : Universitetstryckeriet.

SJUDBJA, 2013 = « СЮДБЯ ХЭТАНЗИ », НАРЬЯНА ВЫНДЕР 16. 14.2.2013.

Online http://nvinder.ru/article/vypusk-no-16-19928-ot-14-fevralya-2013-g/80-syudbya-hetanzi TEREŠČHENKO N. M., 1982 = ТЕРЕЩЕНКО, Н.М., « НЕКОТОРЫЕ ОСОБЕННОСТИ ЯЗЫКА ФОЛЬКЛОРА НЕНЦЕВ », СОВЕТСКОВ ФИННО-УГРОВЕДЕНИЕ XVI, 41-45.

TUISKU Tuula, 1999, Nenetsien ankarat elämisen ehdot tundralla ja kylässä, Rovaniemi : Lapin yliopisto. TUISKU Tuula, 2001, “The Displacement of Nenets Women from Reindeer Herding and the Tundra in the Nenets Autonomous Okrug, Northwestern Russia", Acta Borealia, 18(2), p. 41-60.

VAHTIN Nikolaj Borisovič, 2001 = ЯЗЫКИ НАРОДОВ СЕВЕРА В ХХ ВЕКЕ. ОЧЕРКИ ЯЗЫКОВОГО СДВИГА, САНКТ-ПЕТЕРБУРГ : ЕВРОПЕЙСКИЙ УИВЕРСИТЕТ В САНКТ-ПЕТЕРБУРГЕ.

WILCE James M., 2009, Crying shame: metaculture, modernity, and the exaggerated death of lament, Malden: Wiley-Blackwell.

YOUNG Katherine Galloway, 1987, Taleworlds and Storyrealms. The phenomenology of Narrative, Hingham, Lancaster, Dordrecht: Kluwer Academic Publishers Group.

\section{NOTES}

1. En russe : « ПРИЕЗЖИЕ », nouveaux venus.

2. Chants épiques narratifs de la tradition nenetse.

3. Chants épiques nenetses plus récents, tournés vers la lamentation.

4. En russe : « ПО РАССКАЗАМ ОСТРОВИТЯН ».

5. Jutaminen signifie le déplacement du troupeau de rennes pour se rendre à un autre endroit de pâturage. 
6. En réalité le nom n'a pas été complètement oublié, car du patronyme du nom usuel de Purpej, Filippovič, on peut déduire que son père s'appelait Filipp. C'est un personnage connu dans les remémorations des habitants de lî̂le, et il serait étrange qu'Ardeev ne connaisse pas les souvenirs le concernant.

7. En russe : « ПУРПЭЙ И ЗОЛОТоЙ ЧЕРВЬ ».

8. En russe : « ОБМАНщИКИ ».

\section{RÉSUMÉS}

La plupart des communautés minoritaires du Nord de la Russie ont connu un changement de langue, passant de leur langue autochtone au russe. De plus, bien d'autres changements, depuis la sédentarisation jusqu'à la transformation du rôle de la religion, se sont produits parmi les citoyens soviétiques. Cet article s'interroge sur la nature de la tradition orale, surtout chamanique, après ces mutations et notamment après la propagande athée dans le Nord, surtout parmi les Nénetses. Il s'appuie aussi bien sur des travaux de terrain faits sur l'île de Kolguev, dans la mer de Barents, que sur des travaux d'archives. Les narrateurs de la tradition ont tendance en même temps à minimiser et à souligner le décalage entre leur narration et la tradition nénetse. Les décalages découlent surtout des cadres de la narration. En même temps, les narrateurs définissent implicitement leur rapport au chamanisme.

Most of the northern minority communities of Russia went through a language shift from their own indigenous languages to Russian. In addition, many other changes, from sedentarisation to the change of the role of religion, took place among Soviet citizens. This article discusses the nature of oral, especially shamanistic, tradition after the language shift and atheistic propaganda in the North, among the Nenets. The material is based both on ethnographic fieldwork on the Island of Kolguyev, Barents Sea, and on archival work. The narrators of tradition tend to both minimize and maximize the gap between their telling and the Nenets tradition. The gaps are mainly created with the frames of the narratives. At the same time, the tellers implicitly define their relationship to shamanism.

Suurin osa Venäjän pohjoisista vähemmistöyhteisöistä vaihtoi myöhäisinä neuvostovuosina oman äidinkielensä venäjään, minkä lisäksi elämäntavoissa tapahtui muitakin suuria muutoksia paikalleen asettumisesta uskonnon aseman muutokseen. Artikkelissa pohditaan, millaista suullinen, erityisesti šamanistinen perinne on kielenvaihdon ja ateistisen propagandan jälkeen nenetsien parissa. Artikkelin aineisto on kerätty etnografisessa kenttätyössä Kolgujevin saarella, Barentsin merellä. Lisäksi käytetään sanomalehti- ja arkistoaineistoja. Perinteen kertojat pyrkivät sekä rakentamaan jatkuvuuksia nenetsinkieliseen perinteeseen että tekemään eroja siihen. Näin he tulevat myös määrittäneeksi omaa suhtautumistaan šamanismiin. Pääasiallinen etäisyyden tai läheisyyden luomisen keino on kerronnan kehystäminen. 
INDEX

Mots-clés : chamanisme, Perestrojka, Sjudbabts, changement de langue, Jarabts, narration, Narjana Vynder, sédentarisation

Index géographique : Nénetses (district autonome), Kolguev (île), Nouvelle-Zemble (île),

Nar'jan-Mar

nomsmotscles Nénetses de la toundra, peuples du Nord, Pomores, Russes, Ukrainiens

Thèmes : oralité

disciplines russe, nénetse de la toundra

Keywords : language change, narration, sedentarisation, shamanism, Russian, Tundra Nenets, Twentieth century (beginning), beginning of the 21st century, Arctic peoples, Pomors, Russians, Ukrainians, folklore

Index chronologique : XXe siècle, XXIe siècle 